\title{
Onychoscopy: An Overview
}

\section{${ }^{1}$ Archana Singal, ${ }^{2}$ Deepak Jakhar}

\begin{abstract}
Onychoscopy is the examination of nail and its components with the help of a dermatoscope. It is a noninvasive, painless, cost-effective, and reproducible office procedure. The enhanced and in vivo magnified visualization offered by onychoscopy helps us to identify various features not seen on naked eye examination. So onychoscopy serves as an interface between histological and clinical examination.
\end{abstract}

Keywords: Dermatoscopy, Dermoscopy, Nailfold capillaroscopy, Onychoscopy.

How to cite this article: Singal A, Jakhar D. Onychoscopy: An Overview. Int J Dermoscop 2017;1(2):41-49.

Source of support: Nil

Conflict of interest: None

\section{INTRODUCTION}

Study of nail diseases until recently was largely restricted to more on clinical examination and microbiological evaluation and less on histopathological examination. The unique anatomy of nail and lack of expertise among clinicians often pose a problem in accurate diagnosis and thus management of nail diseases. Surgical intervention on nail unit including diagnostic biopsy is often perceived as a painful procedure. In addition, reluctance of most clinicians to perform biopsies for diagnosis and lack of expertise among pathologists in interpreting the nail histopathology further compound the problem. ${ }^{1}$

The application of dermatoscopy/dermoscopy for the examination of nail has opened an exciting front. ${ }^{2}$ Dermoscopy of nail unit is referred to as onychoscopy. It is a noninvasive and rapid office-based technique applied for the better understanding of nail pathology. ${ }^{2}$ Short learning curve, cost-effectiveness, and reproducibility are other distinct advantages of onychoscopy. It supports retaining photographic documentation and thus is useful for future

\footnotetext{
${ }^{1}$ Director and Professor, ${ }^{2}$ Senior Resident

${ }^{1,2}$ Department of Dermatology and STD, University College of Medical Sciences and Guru Teg Bahadur Hospital, University of New Delhi, Delhi, India

Corresponding Author: Archana Singal, Director and Professor Department of Dermatology and STD, University College of Medical Sciences and Guru Teg Bahadur Hospital University of New Delhi, Delhi, India, Phone: +911122590495 , e-mail: archanasingal@hotmail.com
}

reference and follow-up. It acts as a link between naked eye examination and histopathology. Onychoscopy was initially employed to study the pigmentary abnormalities of nail unit and proximal nail fold capillaries (NFC). However, at present, onychoscopy has fast expanding indications and is an upcoming tool employed in the diagnosis of infective, inflammatory, and neoplastic nail diseases. ${ }^{3-6}$ This article is an attempt to sensitize dermatologists with applications of onychoscopy for improving the diagnosis of various nail diseases and evaluation of treatment outcome.

\section{INSTRUMENTS AND INTERFACE MEDIUM}

As in dermoscopy of the skin, contact or noncontact dermatoscopes can be used for onychoscopy. Both polarized and nonpolarized light sources are essential. At present, there is no consensus on the superiority of one device and light source over another. ${ }^{7}$ Whereas a nonpolarized light source is used for the study of surface anatomy and associated changes (Fig. 1A), polarized light is better suited to study the pigmentation and vascularity (Fig. 1B). It is prudent to start with a lower magnification $(\sim 10 \times)$ for a global view of the pathology and then move to the higher magnification ( $50-200 \times)$ for finer details.

Application of interface medium improves the visualization of color changes and vascularity, especially in the study of proximal nail fold (PNF) capillaries. Various interface medium may be used in onychoscopy, viz. immersion oil, ultrasound gel, and antiseptic alcoholic solutions. ${ }^{8,9}$ However, for examination of the nail apparatus, ultrasound gel is considered to be superior to
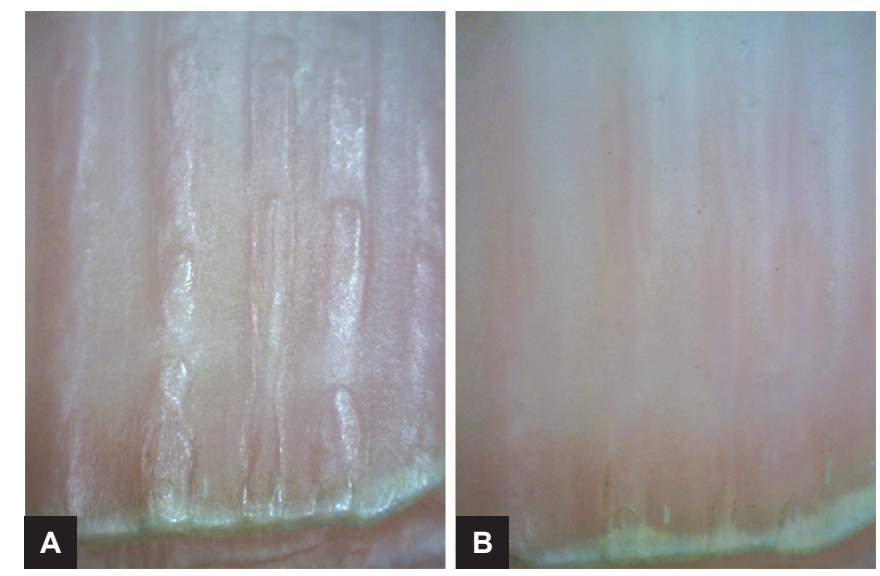

Figs $1 \mathrm{~A}$ and $\mathrm{B}$ : (A) Nonpolarizing onychoscopy showing the surface irregularities (50x); (B) Polarizing onychoscopy showing the nail bed vasculature. Note disappearance of surface irregularities $(50 \times)$ 


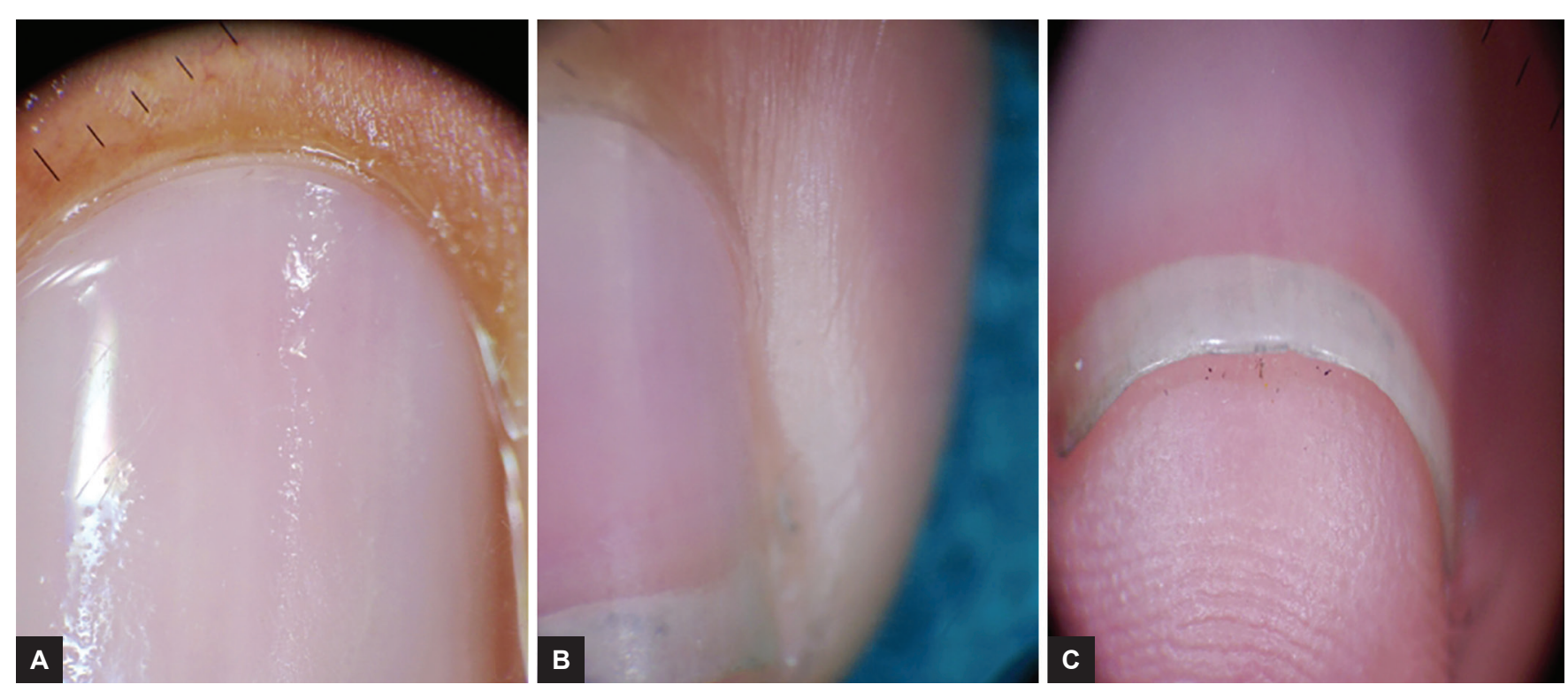

Figs 2A to C: (A) Healthy nail onychoscopy. Note the faintly visible regularly arranged capillaries and shiny and translucent nail plate (10x); (B) lateral nail fold onychoscopy (10x); and (C) onychoscopy of the hyponychium showing the normal ridge pattern (10x)

alcohol because the gel's viscosity prevents it from rolling off the convex nail surface. In our opinion, the choice of interface medium solely depends on individual experience and comfort.

\section{HEALTHY NAIL}

Before recognizing the various pathological signs in the nail unit, it is important to understand onychoscopic features of healthy nail. The PNF shows regularly and evenly spaced hairpin loop vessels, the cuticle perfectly adheres to the nail plate, and the nail plate appears as shiny and translucent structure (Fig. 2A). The underlying pink color represents the nail bed and longitudinal capillaries can be visible in the distal nail bed just proximal to the subcorneal band. Nail matrix can only be visible as lunula. The lateral nail fold and the hyponychium show the normal ridge pattern (Figs 2B and C).

\section{INDICATIONS}

The initial application of onychoscopy was to study the pigmentary abnormalities of nail unit and NFCs in the PNF. In the last one decade, its use has expanded remarkably to include tumors, inflammatory and infective conditions of the nail unit.

\section{Pigmentary Conditions}

\section{Longitudinal Pigmentary Bands}

The most extensively studied nail condition by onychoscopy is melanonychia. The role of onychoscopy in differentiating a benign longitudinal pigmentary band from a malignant one is well established. ${ }^{10-14}$ In 2013, the
International study group on melanonychia published its guidelines for the use of dermoscopy in detection and management of nail pigmentation. ${ }^{7}$

- The first and essential step is to differentiate blood from melanin. ${ }^{7}$ A subungual hematoma is identified by globules of various sizes, usually but not essentially accompanied by distal streaks. The color of globules can range from bright red, brown to black depending upon the depth and duration of hemorrhage (Figs 3 and 4). It is very important to remember that subungual hemorrhage does not rule out melanoma. ${ }^{7}$

- The next step is to differentiate between a benign melanonychia from a malignant melanoma. Benign melanonychia can result from either melanocyte activation or proliferation. A homogeneous graycolored band with thin longitudinal gray lines and

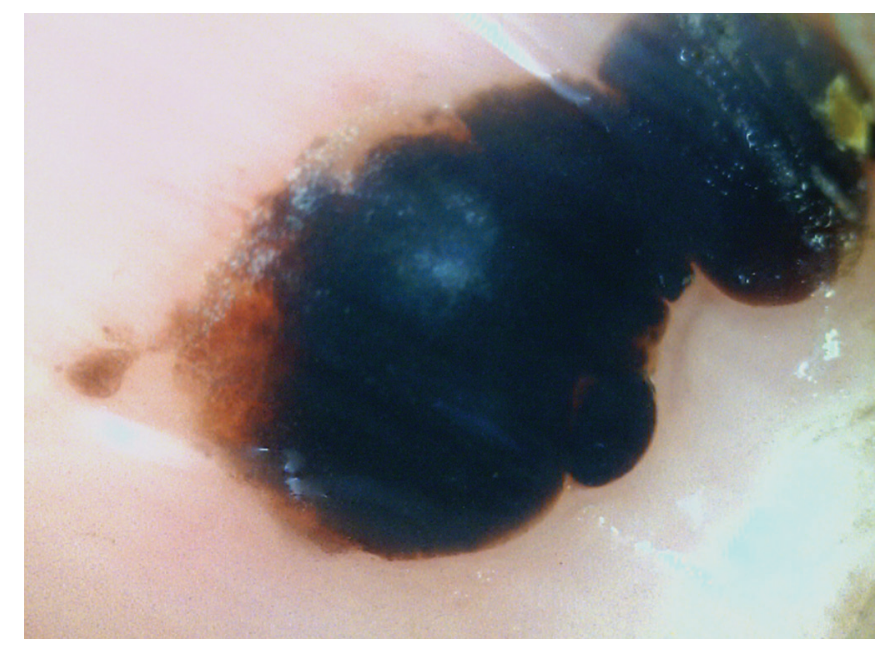

Fig. 3: Onychoscopy of subungual hematoma visible as globules of various sizes 


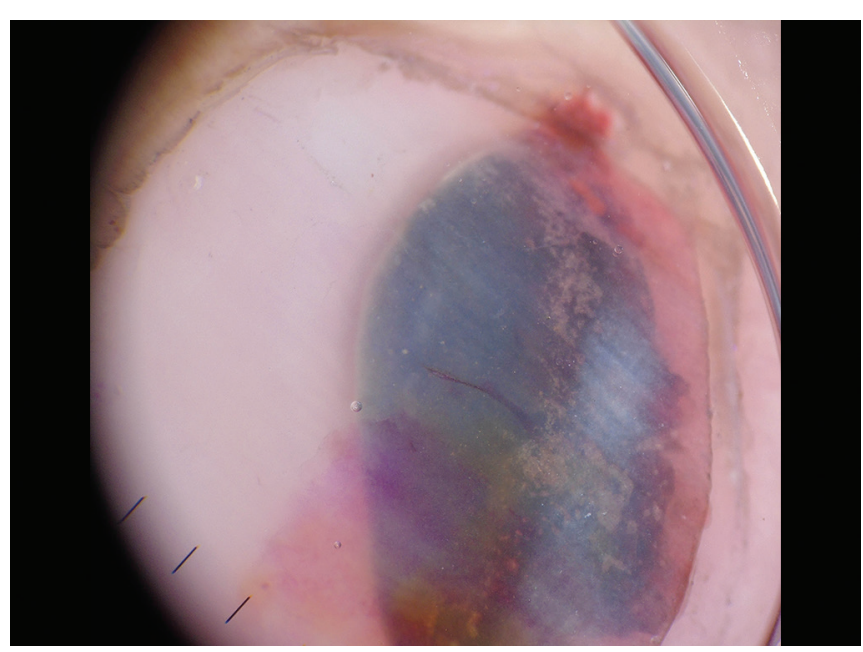

Fig. 4: Onychoscopy of subungual hematoma visible as globules of various colors (10x)

its simultaneous presence in multiple nails indicate melanocyte activation (ethnic or drug induced). It is to be noted that the color and its intensity can vary depending upon the location of pigmentation and nail plate thickness. Melanocyte proliferation as seen in a nevus shows presence of a brown background with regular arrangement of parallel lines (Fig. 5). These parallel longitudinal lines are homogeneous in color, spacing, thickness, and orientation (known as regular pattern). However, another point to be kept in mind is that this regular pattern may not be observed, especially in children and occasionally in adults too. ${ }^{7}$

- Malignant melanoma on the contrary shows longitudinal lines that are irregular in color, spacing, thickness, and parallelism (irregular pattern). Sometimes melanoma may just show diffuse dark background with barely visible lines. The variability in hues of

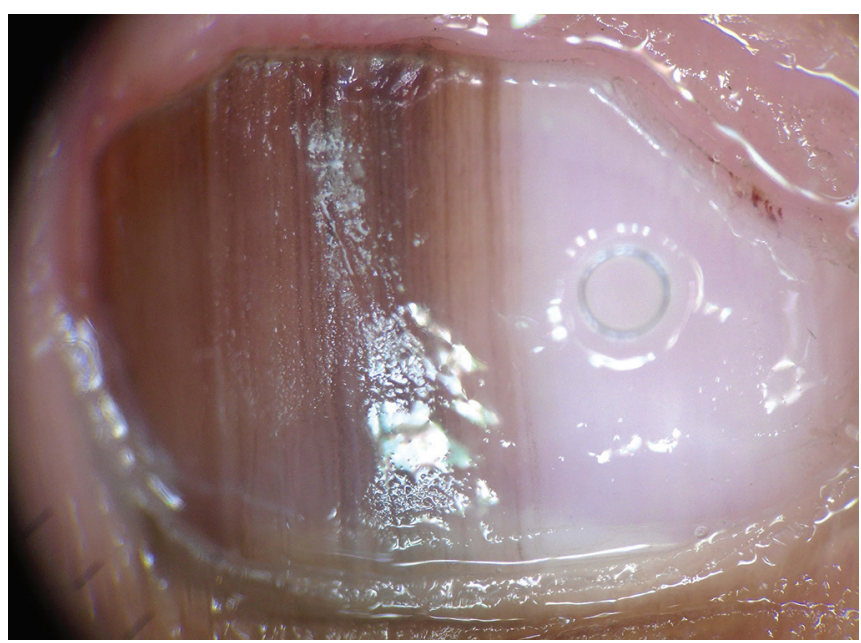

Fig. 5: Onychoscopy of longitudinal melanonychia showing presence of a brown background with regular arrangement of parallel lines indicating melanocyte proliferation. Histopathology confirmed the presence of nevus (10x) pigmentation in such cases may indicate the possibility of melanoma. ${ }^{7}$ Presence of pigmentation in the PNF and/or hyponychium (Hutchinson's sign) also indicates melanoma.

- Onychoscopy of the distal nail plate also serves as a useful tool to determine the nail pigment origin. ${ }^{14}$ Presence of pigment band in the dorsal nail plate indicates its origin in the proximal nail matrix, whereas presence of pigment band in the ventral nail plate indicates its origin in the distal nail matrix. Presence of pigment band throughout the nail plate indicates origin of pigment from both proximal and distal matrix (Fig. 6). ${ }^{14}$

\section{Leukonychia}

Onychoscopy shows presence of longitudinal and/or transverse white streaks. ${ }^{15}$ A transverse white streak originating from the lunula is an early sign of disease in Hailey-Hailey disease. ${ }^{16}$

\section{Inflammatory Diseases}

\section{Nail Psoriasis}

Onychoscopy has markedly improved the visualization of features in nail psoriasis. ${ }^{17,18}$ Onychoscopic features observed depend upon the part of the nail unit affected.

\section{Nail matrix}

- Pits appear as large, deep, and irregular depressions (Fig. 7). ${ }^{17-19}$

- Leukonychia appears as white irregular areas within the nail plate. ${ }^{18}$

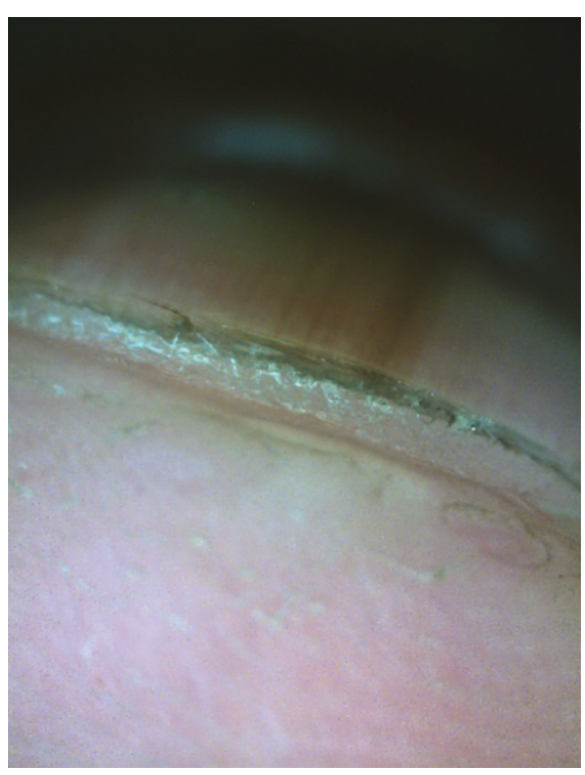

Fig. 6: Onychoscopy of the distal nail plate showing presence of pigment band throughout nail plate indicating origin of pigment from both proximal and distal matrix (10x) 


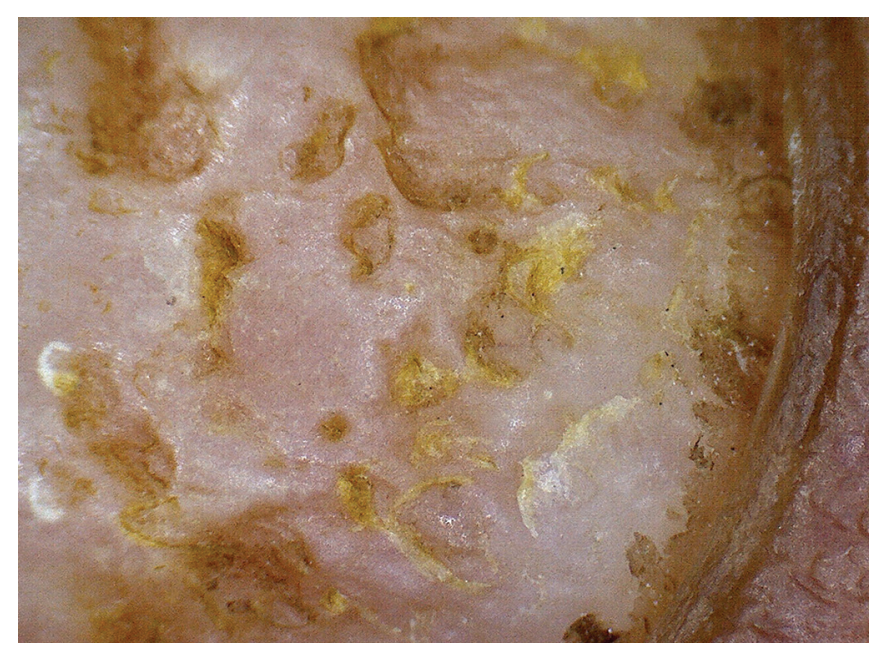

Fig. 7: Psoriatic coarse and irregular pits, some covered with scales $(10 \times)$

\section{Nail bed}

- Salmon patch appears as red to orange-colored patch with irregular shape and size (Fig. 8). ${ }^{17-19}$

- Splinter hemorrhage appears as longitudinal fusiform streaks ranging from bright red to black in color. ${ }^{17-19}$

- Onycholysis: the presence of erythematous border at the proximal end of onycholytic band and absence of longitudinal striae help differentiate psoriasis from onychomycosis (OM; Fig. 8). ${ }^{17-19}$

- Nail bed vessels: Fusiform dilated vessels surrounded by a prominent halo are seen just proximal to the onychodermal band. ${ }^{17}$

- Nail bed hyperkeratosis: Another distinct feature of nail psoriasis is hyperkeratosis of the nail bed (Fig. 9).

Proximal nail fold: Mean capillary is reduced and capillary architecture alterations in the form of dropouts and coiled capillaries are seen. ${ }^{20-23}$

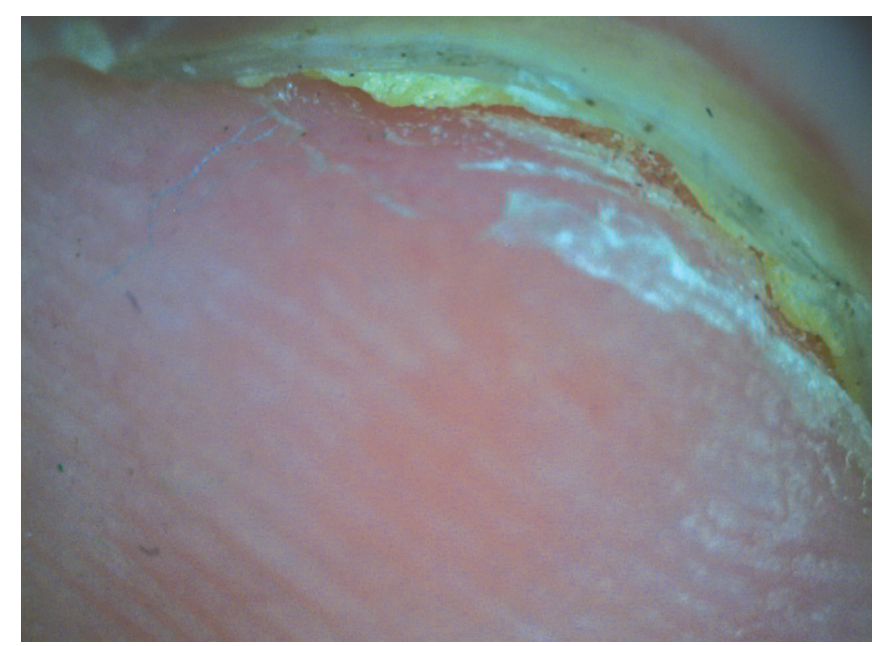

Fig. 9: Onychoscopy of hyponychium showing subungual hyperkeratosis $(10 x)$

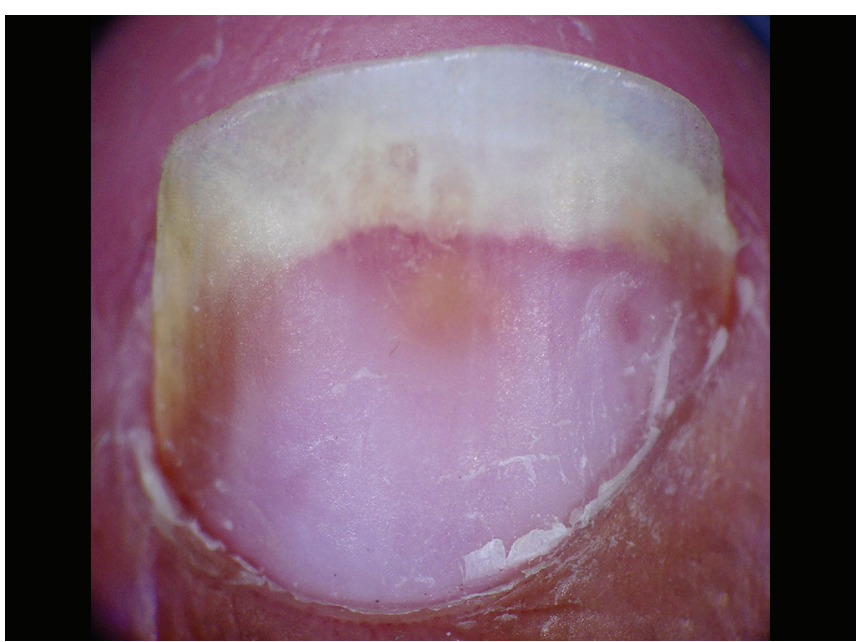

Fig. 8: Onychoscopy of nail psoriasis showing distal onycholysis with proximal erythematous band. Note the presence of salmon spot (10x)

Hyponychium: Dilated and tortuous capillaries may be seen that correlate with the disease severity. ${ }^{24}$

Among the onychoscopic features, nail pitting, splinter hemorrhages and onycholysis are the most common findings. ${ }^{17,18}$

\section{Nail Lichen Planus}

Around $10 \%$ of cases of lichen planus have nail involvement. ${ }^{25}$ As in nail psoriasis, the onychoscopic features observed depend on the part of nail unit affected:

- Nail matrix: Trachyonychia, pitting, pterygium and red lunula (Fig. 10). ${ }^{4,25}$

Nail bed: Chromonychia, nail fragmentation, splinter hemorrhages, onycholysis, subungual keratosis, longitudinal streaks. ${ }^{4,25}$

PNF capillaries: No changes ${ }^{25}$

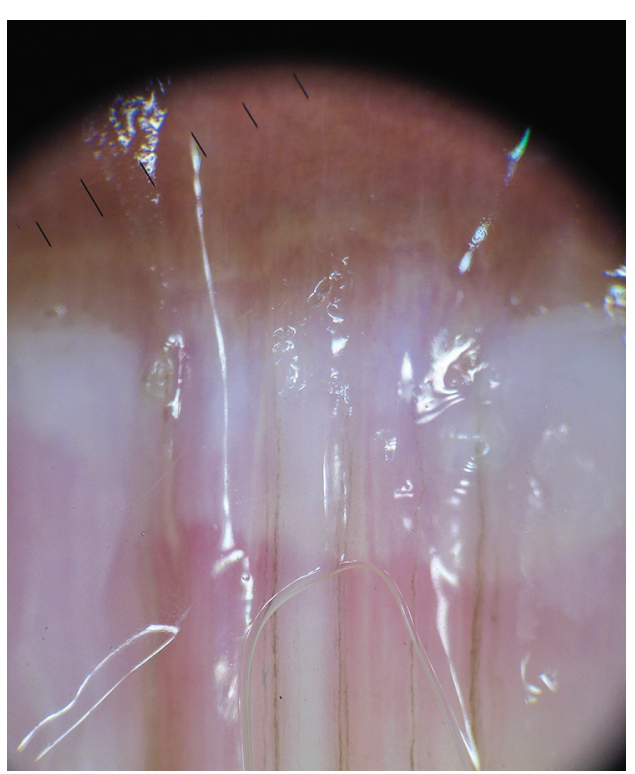

Fig. 10: Onychoscopy of nail lichen plans showing presence of dorsal pterygium $(10 \times)$ 


\section{Infections}

Onychomycosis: The presence of jagged edges with spikes at the proximal edge of onycholytic band corresponds to the fungal invasion of the nail plate (Fig. 11). ${ }^{26-28}$ Onychoscopy is useful in differentiating the pattern of onycholysis seen in OM from nail psoriasis and traumatic onycholysis (Fig. 12). ${ }^{4}$ In contrast to the jagged proximal onycholytic edge in OM (14), it is linear in psoriasis (Fig. 12B), and in traumatic onycholysis linear proximal edge is often associated with subungual hematoma (Fig. 12C). This feature of jagged edge is most commonly but not exclusively seen in OM."Aurora borealis pattern" corresponds to the presence of same or different colors in the onycholytic nail plate (Fig. 13) ${ }^{26}$ Another pattern known as "Ruins aspect" corresponds to the indented areas on the subungual keratosis and distal pulverization (Fig. 14). ${ }^{28}$

Fungal melanonychia is seen as a black to brown longitudinal or transverse band. The typical feature is the

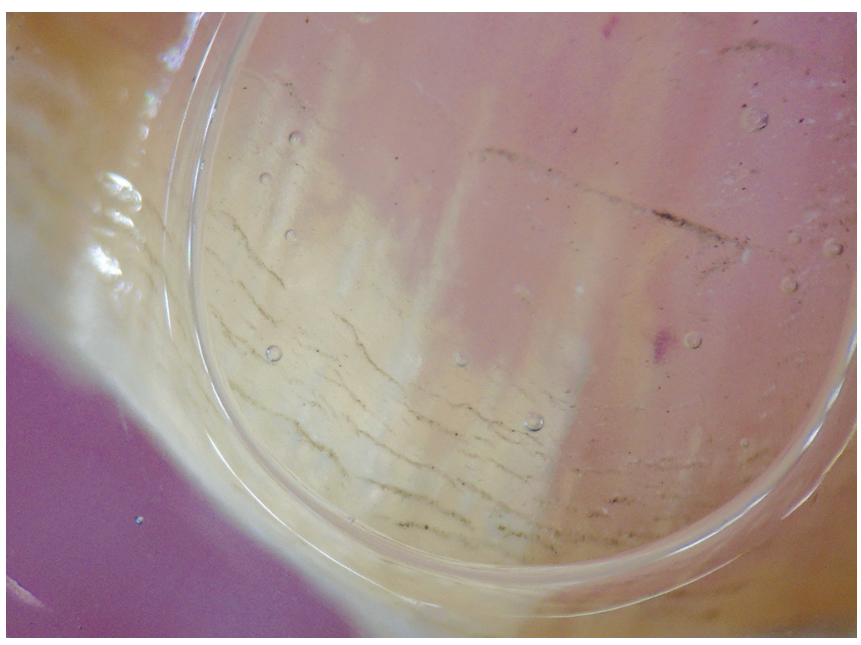

Fig. 11: Jagged proximal edge of onycholytic nail plate in OM (10x) presence of pigment clumps and/or granules within the bands. This corresponds to the fungal colonies. ${ }^{29}$

Periungual warts: Onychoscopy shows presence of thrombosed vessels visible as red to black dots within a hyperkeratotic lesion (Fig. 15). ${ }^{15}$ Onychoscopy in addition helps identify the extent of wart beneath the nail plate (Fig. 15).

\section{Tumors}

Subungual glomus tumor: Typical onychoscopic features have been described for this benign vascular hamartoma arising from the smooth muscle cells of glomus body. ${ }^{30-34}$ Onychoscopy of the nail plate reveals presence of irregular bluish patch with discrete linear vascular structures (Fig. 16). ${ }^{32}$ Intraoperative onychoscopy after removal of nail plate has shown presence of ramified capillaries over a blue background. ${ }^{30}$ This is typically useful for determining the tumor margins as these ramified capillaries disappear abruptly at the margins. ${ }^{30,31}$ "Pink glow" indicating the vascular nature of the tumor has been described with the use of ultraviolet light dermoscopy. ${ }^{34}$

\section{Onychopapilloma}

Onychoscopy of this benign tumor of nail bed and distal matrix shows red bands originating from the lunula with or without splinter hemorrhages. ${ }^{35}$ The band typically shows proximal convex border. Onychoscopy of the distal edge of nail plate shows a characteristic keratotic subungual mass. ${ }^{35}$

\section{Onychomatricoma}

A study of 34 cases of onychomatricoma described onychoscopic features as parallel lateral edges (96.9\%), nail pitting $(93.9 \%)$, thickening of free edge $(93.9 \%)$, dark dots
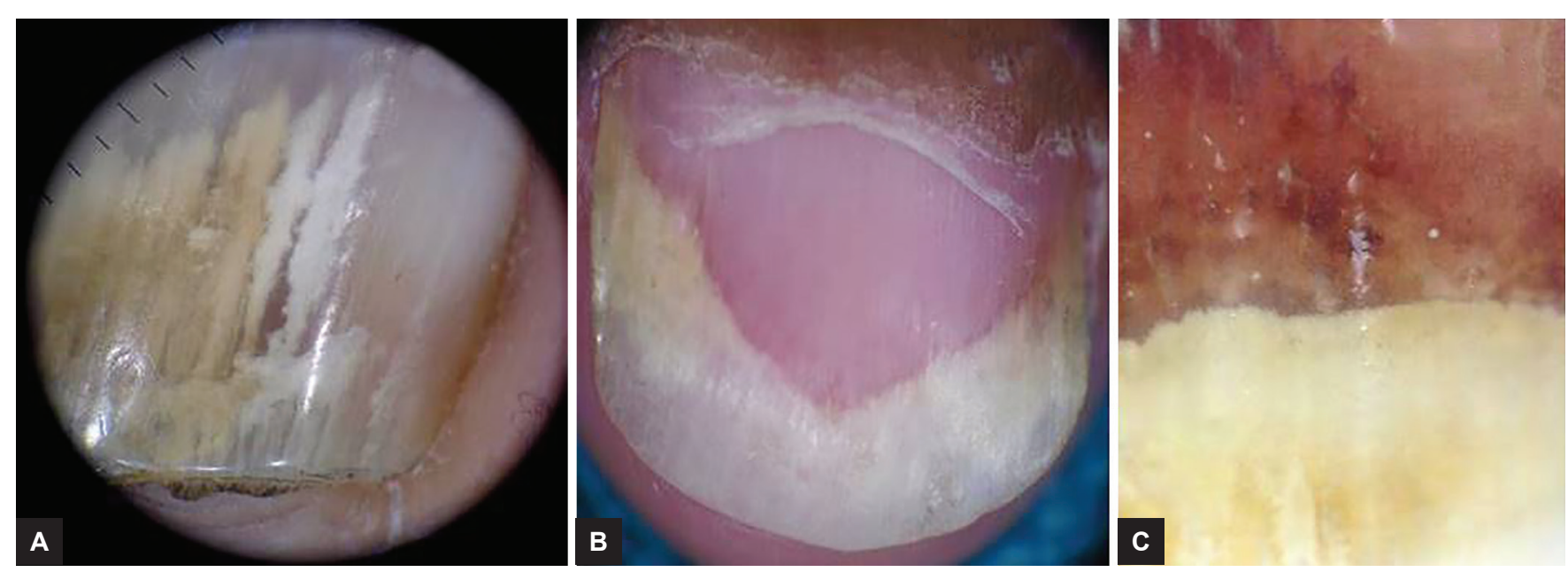

Figs 12A to C: (A) Onychoscopy of OM showing the presence of jagged edges at the proximal end of onycholysis (10x). (B) Onychoscopy of nail psoriasis showing onycholysis with proximal erythematous band (10x). (C) Onychoscopy of traumatic onycholysis. Note the linearity of onycholysis with presence of subungual hematoma (10x) 


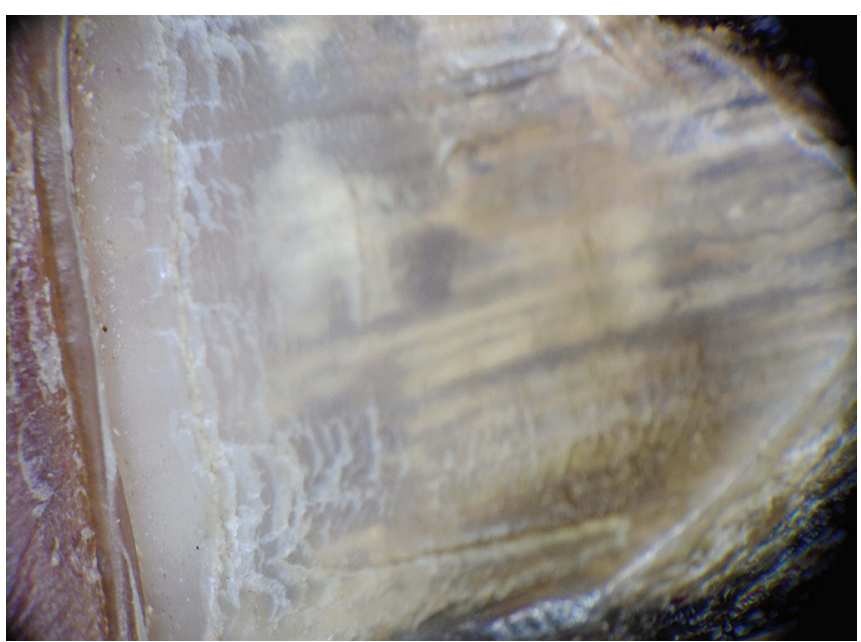

Fig. 13: Different colors; creamy white, yellow, and brown in onycholytic nail plate in OM $(10 \times)$

(84.8\%), splinter hemorrhages (81.8\%), and longitudinal parallel white bands $(81.8 \%)$. As compared with the clinical features, onychoscopic features were found to have less interobserver variations. ${ }^{36}$

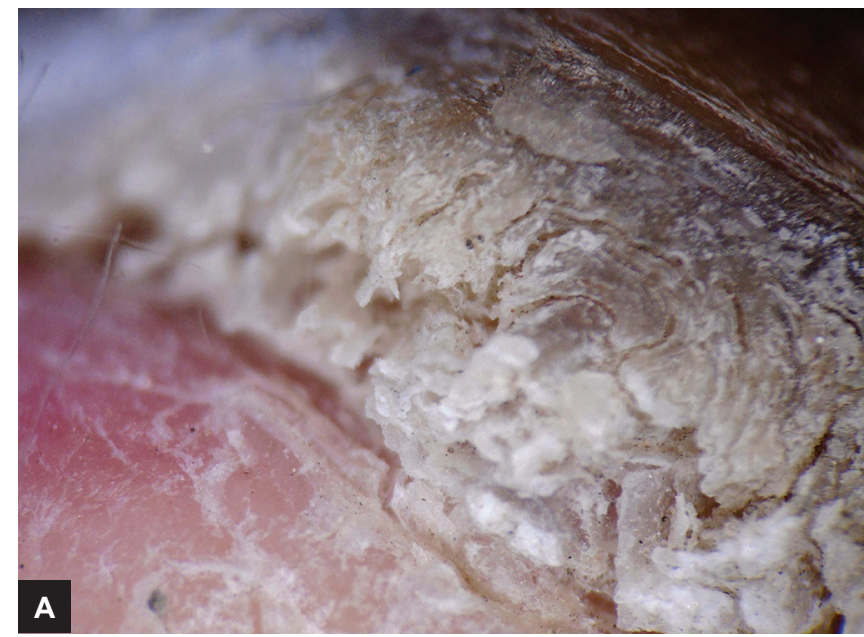

Figs 14A and B: Subungual keratosis with "ruins aspect" (10x)

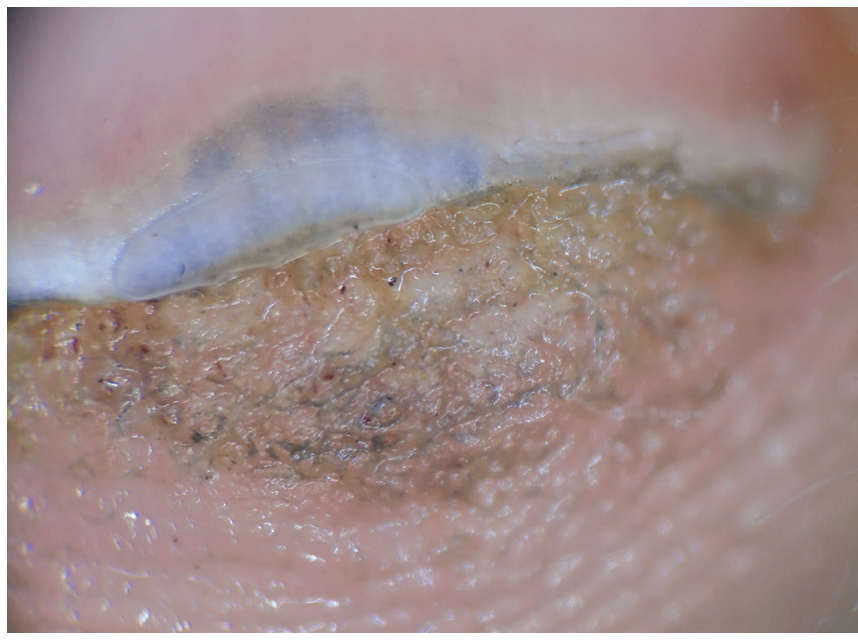

Fig. 15: Onychoscopy of periungual wart with thromboses vessels visible as red dots. Note the extension of wart beneath nail plate, a distinct advantage offered by onychoscopy (10x).

\section{Periungual Bowen's Disease}

Onychoscopy shows presence of white scales and diffusely distributed dotted vessels. ${ }^{37,38}$ The characteristic feature is the presence of whitish halo around these vessels. ${ }^{38}$ The glomerular vessels seen in Bowen's disease at other body sites are typically absent. Another not so common feature is periungual pigmentation, which is described as pseudo-Hutchinson sign. ${ }^{39}$ Pseudo-Hutchinson sign in onychoscopy is described in two other settings: Nail bed hyperpigmentation reflecting through transparent nail folds and nail matrix hyperpigmentation reflecting through transparent nail folds. ${ }^{39}$

\section{Digital Fibrokeratoma}

Clumps of homogeneous red lacunae divided by white meshwork like septal wall are visible on onychoscopy. Telangiectasias are also reported on the adjacent skin (Fig. 17). ${ }^{40}$
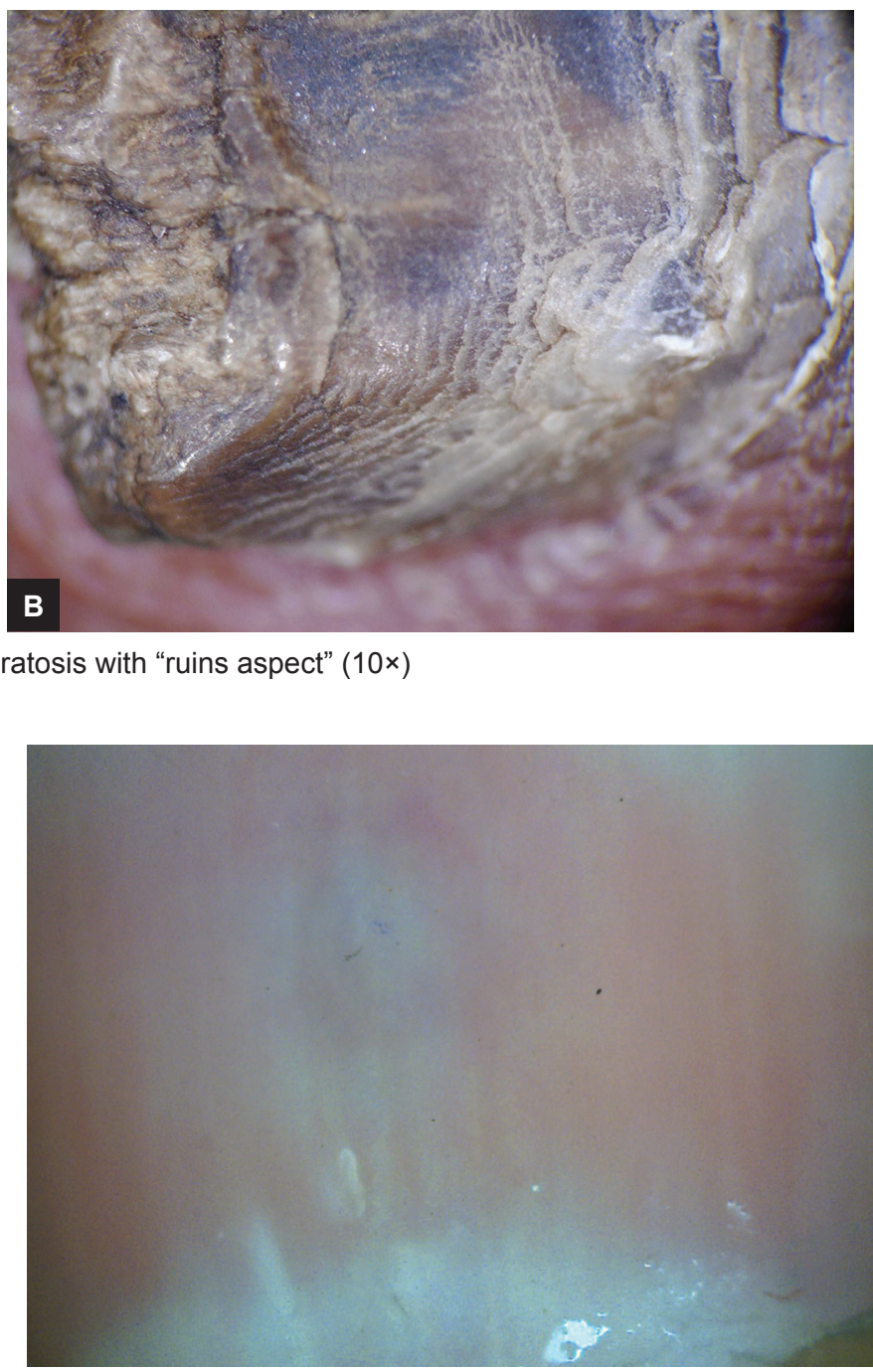

Fig. 16: Onychoscopy of glomus tumor visible as ill-defined bluish patch $(10 \times)$ 


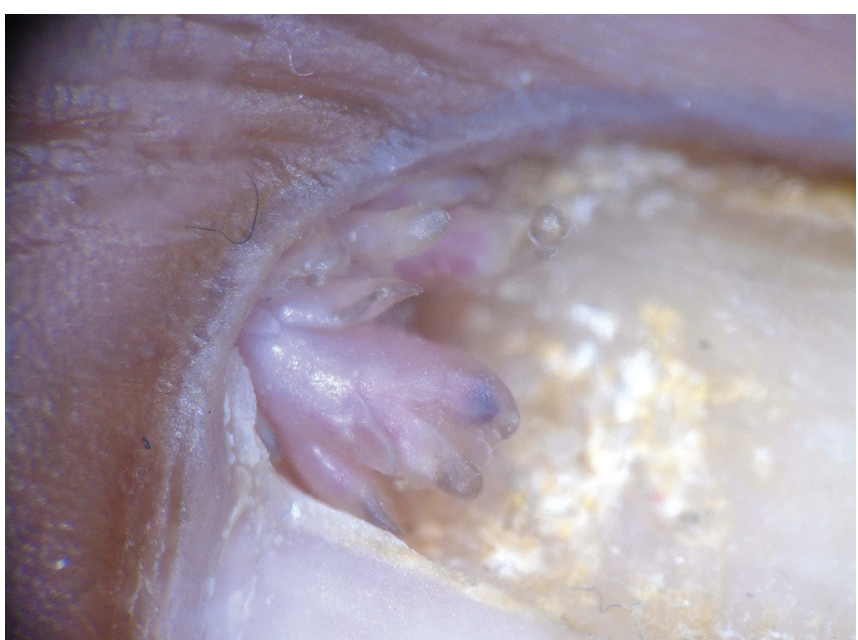

Fig. 17: Onychoscopy of koenen's tumor showing presence of clumps of homogeneous reddish lacunae (10x)

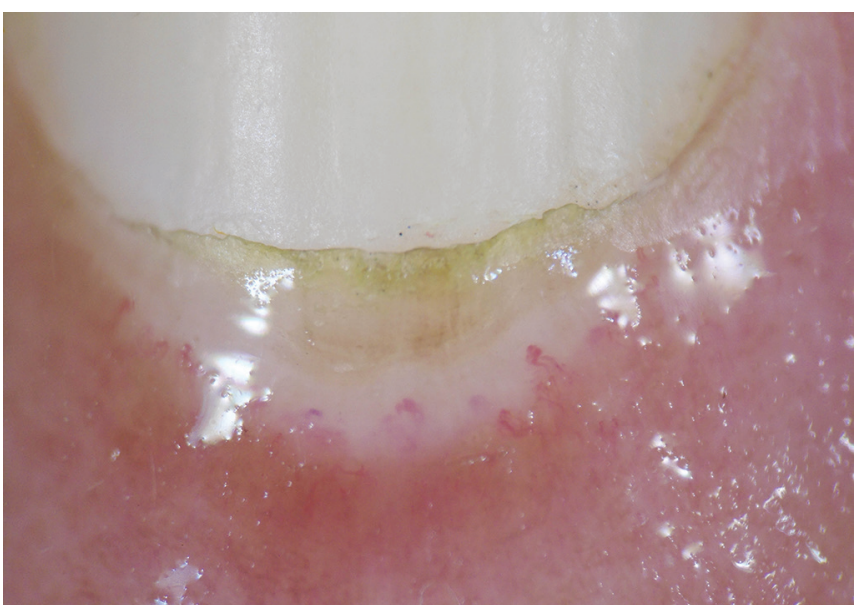

Fig. 19: NFC of "active pattern" in SSc showing derangement of capillaries, a number of giant capillaries, and moderate loss of capillaries $(10 \times)$

\section{Periungual Pyogenic Granuloma}

As seen at other body sites, reddish homogeneous areas with white rail lines are the typical onychoscopic features. ${ }^{41}$

\section{Digital Myxoid Cyst}

When no pressure is applied on myxoid cyst while doing onychoscopy, it shows a vascular pattern in the form of linear, branched, or serpentine vessels. Application of pressure blanches the vascular pattern and the lesion only shows translucent bright white areas. ${ }^{42,43}$

\section{Nailfold Capillaroscopy)}

Nailfold capillaroscopy is a noninvasive imaging technique used for the in vivo assessment of microcirculation. ${ }^{44,45}$ Nailfold capillaroscopy as of now is an established method to assess microcirculation in patients with Raynaud's phenomenon and connective tissue diseases (CTDs), as well as in the early diagnosis and monitoring of systemic sclerosis (SSc).

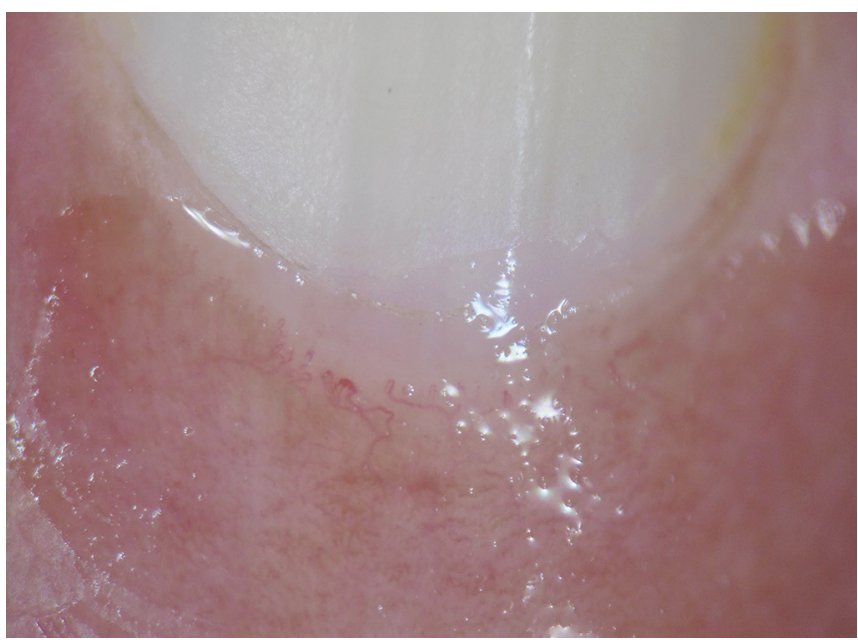

Fig. 18: NFC of "early pattern" in SSc showing few dilated capillaries. Note absence of avascular areas (10x)

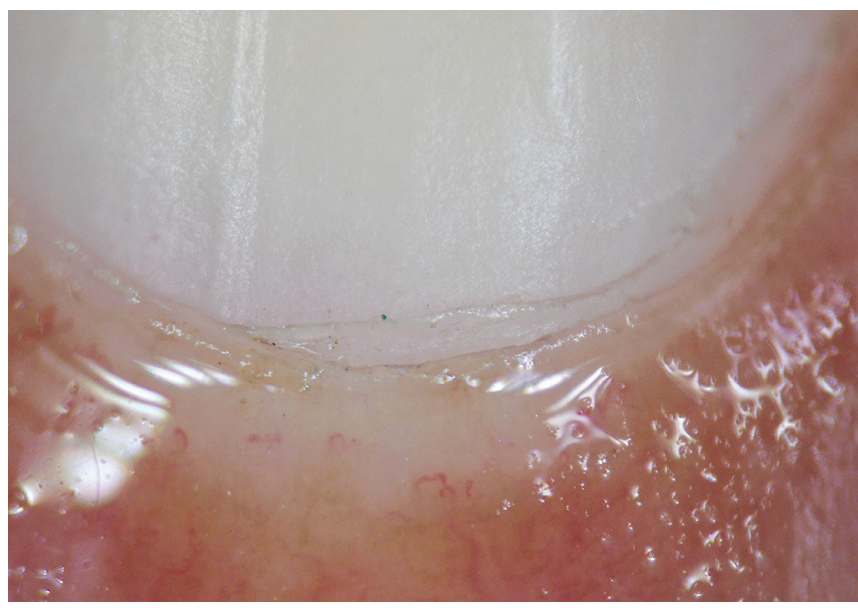

Fig. 20: NFC of "late pattern" in SSc showing avascular areas indicating severe loss of capillaries (10x)

\section{Sytemic Sclerosis}

Three different patterns of NFC changes have been described with SSc: ${ }^{46}$

- Early pattern: Appearance of few dilated and/or giant capillaries and a few hemorrhages. Capillary distribution is relatively preserved without loss of capillaries (Fig. 18).

- Active pattern: Large numbers of giant capillaries, hemorrhages, a moderate loss of capillaries, slight derangement, and diffuse pericapillary edema can be found (Fig. 19).

- Late pattern: Severe loss of capillaries with extensive avascular areas, bushy and ramified capillaries, or more than one capillary loop in a dermal papilla (Fig. 20).

\section{Dermatomyositis (DM) Polymyositis (PM)}

Dermatomyositis is only the second CTD that shows the most consistent NFC abnormalities. The pattern observed in DM resembles SSc to a large extent and includes the 
presence of two or more of the following characteristics in two or more nail folds, viz. capillary dilation, twisted enlarged capillaries, loss of capillaries, bushy capillaries, disorganization of capillary architecture, and microhemorrhages. $^{47,48}$

Capillary dilation and loss of capillaries are more commonly and severely reported in DM as compared with PM. ${ }^{47}$

\section{Other CTDs}

Systemic lupus erythematosus, rheumatoid arthritis, mixed CTD, undifferentiated CTD, and Sjögren's syndrome are known to show capillaroscopic changes. ${ }^{49}$

Onychoscopy has become an established and important diagnostic tool for nail disorders with fast expanding indications. The onychoscopic criteria for majority of the inflammatory and infective nail diseases are evolving at a fast pace. With an increasing interest in the so far neglected nail disorders, it has become imperative for all clinicians to be familiar with the science of onychoscopy to supplement their primary clinical skills.

\section{REFERENCES}

1. Grover C, Chaturvedi UK, Reddy BS. Role of nail biopsy as a diagnostic tool. Indian J Dermatol Venereol Leprol 2012 May-Jun;78(3):290-298.

2. Lencastre A, Lamas A, Sá D, Tosti A. Onychoscopy. Clin Dermatol 2013 Sep-Oct;31(5):587-593.

3. Ronger S, Touzet S, Ligeron C, Balme B, Viallard AM, Barrut D, Colin C, Thomas L. Dermoscopic examination of nail pigmentation. Arch Dermatol 2002 Oct;138(10):1327-1333.

4. Nakamura RC, Costa MC. Dermatoscopic findings in the most frequent onychopathies: descriptive analysis of 500 cases. Int J Dermatol 2012 Apr;51(4):483-496.

5. Mannarino E, Pasqualini L, Fedeli F, Scricciolo V, Innocente S. Nailfold capillaroscopy in the screening and diagnosis of Raynaud's phenomenon. Angiology 1994 Jan;45(1):37-42.

6. Blockmans D, Beyens G, Verhaeghe R. Predictive value of nailfold capillaroscopy in the diagnosis of connective tissue diseases. Clin Rheumatol 1996 Mar;15(2):148-153.

7. Di Chiacchio ND, Farias DC, Piraccini BM, Hirata SH, Richert B, Zaiac M, Daniel R, Fanti PA, Andre J, Ruben BS, et al. Consensus on melanonychia nail plate dermoscopy. An Bras Dermatol 2013 Mar-Apr;88(2):309-313.

8. Tasli L, Oguz O. The role of various immersion liquids at digital dermoscopy in structural analysis. Indian J Dermatol Venereol Leprol 2011 Jan-Feb;77(1):110.

9. Gewirtzman AJ, Saurat JH, Braun RP. An evaluation of dermoscopy fluids and application techniques. Br J Dermatol 2003 Jul;149(1):59-63.

10. Haas N, Henz BM. Pitfall in pigmentation: pseudopods in the nail plate. Dermatol Surg 2002 Oct;28(10):966-967.

11. Imakado $S$, Sato $H$, Hamada $K$. Two cases of subungueal melanoma in situ. J Dermatol 2008 Nov;35(11):754-758.

12. Antonovich DD, Grin C, Grant-Kels JM. Childhood subungual melanoma in situ in diffuse nail melanosis beginning as expanding longitudinal melanonychia. Pediatr Dermatol 2005 May-Jun;22(3):210-212.

13. Iorizzo M, Tosti A, Di Chiacchio N, Hirata SH, Misciali C, Michalany N, Domiguez J, Toussaint S. Nail melanoma in children: differential diagnosis and management. Dermatol Clin 2008 Jul;34(7):974-978.

14. Braun RP, Baran R, Saurat JH, Thomas L. Surgical Pearl: dermoscopy of the free edge of the nail to determine the level of nail plate pigmentation and the location of its probable origin in the proximal or distal nail matrix. J Am Acad Dermatol 2006 Sep;55(3):512-513.

15. Piraccini BM, Bruni F, Starace M. Dermoscopy of non skin cancer nail disorders. Dermatol Ther 2012 Nov-Dec;25(6):594 602.

16. Bel B, Jeudy G, Vabres P. Dermoscopy of longitudinal leukonychia in Hailey-Hailey disease. Arch Dermatol 2010 Oct;146(10):1204.

17. Yadav TA, Khopkar US. Dermoscopy to detect signs of subclinical nail involvement in chronic plaque psoriasis: a study of 68 patients. Indian J Dermatol 2015 May-Jun;60(3):272-275.

18. Yorulmaz A, Artuz F. A study of dermoscopic features of nail psoriasis. Postepy Dermatol Alergol 2017 Feb;34(1):28-35.

19. Farias DC, Tosti A, Chiacchio ND, Hirata SH. Dermoscopy in nail psoriasis. An Bras Dermatol 2010 Jan-Feb;85(1):101-103.

20. Ohtsuka T, Yamakage A, Miyachi Y. Statistical definition of nailfold capillary pattern in patients with psoriasis. Int J Dermatol 1994 Nov;33(11):779-782.

21. Zaric D, Clemmensen OJ, Worm AM, Stahl D. Capillary microscopy of the nail fold in patients with psoriasis and psoriatic arthritis. Dermatologica 1982 Jan;164(1):10-14.

22. Bhushan M, Moore T, Herrick AL, Griffiths CEM. Nailfold video capillaroscopy in psoriasis. Br J Dermatol 2000 Jun;142(6):1171-1176.

23. Ribeiro CF, Siqueira EB, Holler AP, Fabrício L, Skare TL. Periungual capillaroscopy in psoriasis. An Bras Dermatol 2012 Jul-Aug;87(4):550-553.

24. Iorizzo M, Dahdah M, Vicenzi C, Tosti A. Videodermoscopy of the hyponychium in nail bed psoriasis. J Am Acad Dermatol 2008 Apr;58(4):714-715.

25. Nakamura R, Broce AA, Palencia DP, Ortiz NI, Leverone A. Dermatoscopy of nail lichen planus. Int J Dermatol 2013 Jun;52(6):684-687.

26. Piraccini BM, Balestri R, Starance M, Rech G. Nail digital dermoscopy (onychoscopy) in the diagnosis of onychomycosis. J Eur Acad Dermatol Venereol 2013 Apr;27(4):509-513.

27. Jesús-Silva MA, FernándezMartínez R, Roldán-Marín $R$, Arenas R. Dermoscopic patterns in patients with a clinical diagnosis of onychomycosis-results of a prospective study including data of potassium hydroxide $(\mathrm{KOH})$ and culture examination. Dermatol Pract Concept 2015 Apr;5(2):39-44.

28. De Crignis G, Valgas N, Rezende P, Leverone A, Nakamura R. Dermatoscopy of onychomycosis. Int J Dermatol 2014 Feb;53(2):e97-e99.

29. Kilinc Karaarslan I, Acar A, Aytimur D, Akalin T, Ozdemir F. Dermoscopic features in fungal melanonychia. Clin Exp Dermatol 2015 Apr;40(3):271-278.

30. Maehara LdeS, Ohe EM, Enokihara MY, Michalany NS, Yamada S, Hirata SH. Diagnosis of glomus tumor by nail bed and matrix dermoscopy. An Bras Dermatol 2010 MarApr;85(2):236-238.

31. Rai AK. Role of intraoperative dermoscopy in excision of nail unit glomus tumor. Indian Dermatol Online J 2016 Sep;7(5):448-450. 
32. Duarte AF, Correia O, Barreiros H, Haneke E. Giant subungual glomus tumor: clinical, dermoscopy, imagiologic and surgery details. Dermatol Online J 2016 Oct;22(10):11.

33. Mutsaers ER, Genders R, van Es N, Kukutsch N. Dermoscopy of glomus tumor: more white than pink. J Am Acad Dermatol 2016 Jul;75(1):e17-e18.

34. ThatteSS, Chikhalkar SB, Khopkar US. "Pink glow": a new sign for thediagnosis of glomus tumor on ultraviolet light dermoscopy. Indian Dermatol Online J 2015 Dec;6(Suppl 1):S21-S23.

35. Tosti A, Schneider SL, Ramirez Quizon MN, Zaiac M, Miteva M. Clinical, dermoscopic, and pathologic features of onychopapilloma: a review of 47 cases. J Am Acad Dermatol 2016 Mar;74(3):521-526.

36. LesortC,DebarbieuxS, DuruG, DalleS, Poulhalon N, Thomas L. Dermoscopic features of onychomatricoma: a study of 34 cases. Dermatology 2015 Jun;231(2):177-183.

37. Nakayama C, Hata H, Homma E, Fujita Y, Shimizu H. Dermoscopy of periungual pigmented Bowen's disease: its usefulness in differentiation from malignant melanoma. J Eur Acad Dermatol Venereol 2016 Mar;30(3):552-554.

38. Giacomel J, Lallas A, Zalaudek I, Argenziano G. Periungual Bowen disease mimicking chronic paronychia and diagnosed by dermoscopy. J Am Acad Dermatol 2014 Sep;71(3):e65-e67.

39. Baran R, Kechijian P. Hutchinson's sign: a reappraisal. J Am Acad Dermatol 1996 Jan;34(1):87-90.

40. Hayashi K, Matori S, Kariya Y, Sonosaki T, Yamaguchi S, Hagiwara K, Uezato H, Takahashi K. Dermoscopic observation of acquired digital fibrokeratoma developed on the dorsum of the fourth left toe. J Dermatol 2016 Jan;43(1):107-108.

41. Yorulmaz A, Yalcin B. A painful periungual red spot in a patient with onychodystrophy. Skin Appendage Disord 2017 May;3(2):67-69.

42. Salerni G, González R, Alonso C. Dermatoscopic pattern of digital mucous cyst: report of three cases. Dermatol Pract Concept 2014 Oct;4(4):65-67.

43. Salerni G, Alonso C. Images in clinical medicine. Digital mucous cyst. N Engl J Med 2012 Apr;366(14):1335.

44. Cortes S, Cutolo M. Capillaroscopic patterns in rheumatic diseases. Acta Reum Port 2007 Jan-Mar;32(1):29-36.

45. Shore AC. Capillaroscopy and measurement of capillary pressure. Br J Clin Pharmacol 2000 Dec;50(6):501-513.

46. Cutolo M, Sulli A, Pizzorni C, Accardo S. Nail fold videocapillaroscopy assessment of microvascular damage in systemic sclerosis. J Rheumatol 2000 Jan;27(1):155-160.

47. Ganczarczyk ML, Lee P, Armstrong SK. Nailfold capillary microscopy in polymyositis and dermatomyositis. Arthritis Rheum 1988 Jan;31(1):116-119.

48. Klyscz T, Bogenschutz O, Junger M, Rassner G. Microangiopathic changes and functional disorders of nail fold capillaries in dermatomyositis. Hautarzt 1996 Apr;47(4):289-293.

49. Cutolo, M. Capillaroscopy in rheumatic diseases from the XVIII to the XXI centry. In: Cutolo M, editor. Atlas of capillaroscopy in rheumatic diseases. Vol. 1. Milano: Elsevier Srl; 2010. p. 3-5. 